\title{
Image Contrast Enhancement by Interpolated Histogram Specification
}

\author{
Hengjun Yu, Kohei Inoue*, Kenji Hara, and Kiichi Urahama \\ Kyushu University, 4-9-1, Shiobaru, Minami-ku, Fukuoka 815-8540, Japan \\ *Corresponding Author: k-inoue@design.kyushu-u.ac.jp
}

\begin{abstract}
We propose a method for enhancing the contrast of a given grayscale image by specifying the interpolated histogram of the equalized one as a target histogram for the histogram specification method. The proposed histogram interpolation method fills empty bins in the equalized histogram by using the neighboring non-zero elements, and preserves the shape of original histogram. As a result, we can avoid the image over-enhancement which frequently occurs in conventional histogram equalization. Experimental results demonstrate the effectiveness of the proposed method visually and quantitatively with objective image quality measures.
\end{abstract}

Keywords: image contrast enhancement, interpolated histogram specification, histogram equalization.

\section{Introduction}

Contrast enhancement (CE) is an important issue in the field of image processing. Histogram equalization (HE) ${ }^{(1)}$ is a well-known method for CE. However, HE tends to overenhance the contrast of low contrast images, and amplify noise ${ }^{(2)}$. As a result, the enhanced images may have false contours. We can find the footprint of the over-enhancement in the histogram of the histogram- equalized image, i.e., the resultant histogram frequently becomes sparse, in other words, a number of elements of the histogram are zero. The sparsity of the histogram indicates that there exist gaps in continuous-tone.

To overcome such a difficulty in HE, we have recently proposed a histogram specification (HS) method ${ }^{(3)}$ based on the fast ordering algorithm by Nikolova and Steidl ${ }^{(4)}$, which bridges the gap by filling the empty bins in the equalized histogram with piecewise constant non-zero values. This HS method $^{(3)}$ achieved better performance than conventional HE for most of the tested images. However, the HS method ${ }^{(3)}$ is computationally expensive compared to $\mathrm{HE}$ because Nikolova's method ${ }^{(4)}$ is a demanding procedure. To reduce the computational costs in the HS method ${ }^{(3)}$, we have also proposed more computationally efficient methods ${ }^{(5)}$ without Nikolova's method ${ }^{(4)}$. However, we have observed that some images are still over-enhanced even if we use the HS methods $^{(3,5)}$.

In this paper, we propose an improved HS method, where we interpolate a sparse histogram by using a procedure for selecting local maximum and minimum values. Experimental results show that the proposed method can suppress the contrast over-enhancement caused by the previous methods.

The rest of this paper is organized as follows. Section 2 describes the proposed interpolated histogram specification. Section 3 summarizes image quality measures used in the experiments. Section 4 shows experimental results. Finally, Section 5 concludes this paper.

\section{Interpolated Histogram Specification}

In this section, we first summarize histogram equalization method, and then propose a histogram interpolation method. After that, we describe a histogram specification method based on the interpolated histogram.

\subsection{Histogram Equalization}

Let $G$ be a grayscale image, and let $g_{i j} \in$ $\{0,1, \ldots, L-1\}$ for $i=1,2, \ldots, m$ and $j=1,2, \ldots, n$ be the intensity at the position $(i, j)$ in $G$, where $L$ denotes the number of intensity levels, e.g., $L=2^{8}=256$ for 8-bit images. Let $\boldsymbol{h}=\left[h_{0}, h_{1}, \ldots, h_{L-1}\right]$ be the histogram of $G$, then the $k$ th element of $\boldsymbol{h}$ is given by $h_{k}=$ $\sum_{i=1}^{m} \sum_{j=1}^{n} \delta_{g_{i j}, k}$ where $\delta_{g_{i j}, k}$ denotes the Kronecker delta such that $\delta_{g_{i j}, k}=1$ if $g_{i j}=k, \delta_{g_{i j}, k}=0$ otherwise. Let $\boldsymbol{H}=\left[H_{0}, H_{1}, \ldots, H_{L-1}\right]$ be the cumulative histogram of $\boldsymbol{h}$, where the $k$ th element of $\boldsymbol{H}$ is given by $H_{k}=\sum_{k^{\prime}=0}^{k} h_{k^{\prime}}$. 
Then the histogram-equalized image $G^{E}=\left[g_{i j}^{E}\right]$ of $G$ is given by

$$
g_{i j}^{E}=\operatorname{round}\left(\frac{L-1}{m n} H_{g_{i j}}\right)
$$

Let $\boldsymbol{h}^{E}=\left[h_{0}^{E}, h_{1}^{E}, \ldots, h_{L-1}^{E}\right]$ be the histogram of $G^{E}$, then, for a low contrast image $G, h^{E}$ frequently becomes sparse, i.e., a number of elements of $\boldsymbol{h}^{E}$ are zero. The sparsity of $\boldsymbol{h}^{E}$ indicates the gaps in continuous-tone, and suggests the occurrence of false contours in $G^{E}$. In the next section, we propose a method for interpolating $\boldsymbol{h}^{E}$ to reduce the empty bins in $\boldsymbol{h}^{E}$.

\subsection{Interpolating Equalized Histogram}

To reduce the empty bins in $\boldsymbol{h}^{E}$, we fill them by interpolation. Let $\boldsymbol{h}^{F}=\left[h_{0}^{F}, h_{1}^{F}, \ldots, h_{L-1}^{F}\right]$ be the interpolated one. We proposed to fill the $k$ th element of $\boldsymbol{h}^{F}$ is given by

$$
h_{k}^{F}=\min \left\{h_{k-1}^{U}, h_{k}^{U}, h_{k+1}^{U}\right\}
$$

where $h_{k}^{U}$ denotes the $k$ th element of $\boldsymbol{h}^{U}=$ $\left[h_{0}^{U}, h_{1}^{U}, \ldots, h_{L-1}^{U}\right]$ defined by

$$
h_{k}^{U}=\max \left\{h_{k-1}^{E}, h_{k}^{E}, h_{k+1}^{E}\right\},
$$

where we define $h_{-1}^{E}=h_{L}^{E}=-\infty$ and $h_{-1}^{U}=h_{L}^{U}=\infty$.

\subsection{Histogram Specification}

Let $\boldsymbol{H}^{F}=\left[H_{0}^{F}, H_{1}^{F}, \ldots, H_{L-1}^{F}\right]$ be the cumulative histogram of $\boldsymbol{h}^{F}$, then the $k$ th element of $\boldsymbol{H}^{F}$ is given by $H_{k}^{F}=\sum_{k^{\prime}=0}^{k} h_{k^{\prime}}^{F}$. Let $G^{F}=\left[g_{i j}^{F}\right]$ be the output image of the histogram specification method with $\boldsymbol{h}^{F}$. Then the pixel value is given by

$$
g_{i j}^{F}=\arg \min _{k}\left\{\left|H_{L-1} H_{k}^{F}-H_{L-1}^{F} H_{g_{i j}}\right|\right\}
$$

The proposed algorithm is summarized as follows:

\footnotetext{
Algorithm 1 Interpolated Histogram Specification

Require: a grayscale image $G=\left[g_{i j}\right]$

Ensure: a contrast-enhanced image $G^{F}=\left[g_{i j}^{F}\right]$

01: Compute the histogram $\boldsymbol{h}$ of $G$;

02: Compute the cumulative histogram $\boldsymbol{H}=$ $\left[H_{0}, H_{1}, \ldots, H_{L-1}\right]$ of $\boldsymbol{h}$;

03: Compute the histogram-equalized image $G^{E}$ of $G$;

04: Compute the histogram $\boldsymbol{h}^{E}$ of $G^{E}$;

05: Compute the interpolated histogram $\boldsymbol{h}^{F}$ in (2) from
}

$$
\boldsymbol{h}^{E} \text {; }
$$

06: Compute the cumulative histogram $\boldsymbol{H}^{F}=$ $\left[H_{0}^{F}, H_{1}^{F}, \ldots, H_{L-1}^{F}\right]$ of $\boldsymbol{h}^{F}$;

07: for $i:=1$ to $m$ do

08: for $j:=1$ to $n$ do

09: $\quad g_{i j}^{F}:=\arg \min _{k}\left\{H_{L-1} H_{k}^{F}-H_{L-1}^{F} H_{g_{i j}}\right\}$;

10: end for

11: end for

\section{Image Quality Measures}

In this section, we summarize three image quality measures: the expected measure of enhancement by gradient $(\mathrm{EMEG})^{(6)}$, the gradient magnitude similarity deviation $(G M S D)^{(7)}$ and the combination of EMEG and GMSD (EMEG/GMSD) $)^{(3,5)}$, which will be used for the performance evaluation of the proposed method in the next section.

\subsection{Expected Measure of Enhancement by Gradient}

EMEG proposed by Celik ${ }^{(6)}$ is defined by

$\operatorname{EMEG}(G)$

$$
=\frac{1}{M N} \sum_{I=1}^{M} \sum_{J=1}^{N} \frac{1}{\beta} \max \left(\frac{G_{I, J}^{d x, h}}{G_{I, J}^{d x, l}+\epsilon}, \frac{G_{I, J}^{d y, h}}{G_{I, J}^{d y, l}+\epsilon}\right),
$$

where $G_{I, J}^{d x, h}\left(G_{I, J}^{d y, h}\right)$ and $G_{I, J}^{d x, l}\left(G_{I, J}^{d y, l}\right)$ are the maximum and minimum values of absolute valued derivatives in horizontal (vertical) direction of $w_{1} \times w_{2}$ sub-block $G_{I, J}$ of an image $G, \beta=L-1$, and $\epsilon$ is a positive constant. We set $\epsilon=1, w_{1}=w_{2}=8, M=\operatorname{fix}\left(m / w_{1}\right)$ and $N=\operatorname{fix}(n /$ $w_{2}$ ) where fix denotes a function for rounding toward zero. The larger the value of EMEG of an image is, the higher the quality of the image is.

\subsection{Gradient Magnitude Similarity Deviation}

GMSD proposed by Xue et al. ${ }^{(7)}$ is a full reference image quality assessment model defined by

$$
\operatorname{GMSD}\left(G, G^{\prime}\right)
$$

$$
=\sqrt{\frac{1}{m n} \sum_{i=1}^{m} \sum_{j=1}^{n}\left(\mathrm{GMS}_{i j}^{G, G^{\prime}}-\mathrm{GMSM}^{G, G^{\prime}}\right)^{2}},
$$


where $\mathrm{GMSM}^{G, G^{\prime}}$ denotes the average of $\mathrm{GMS}_{i j}^{G, G^{\prime}}$ which is the value of the GMS map at $(i, j)$ defined by

$$
\mathrm{GMS}_{i j}^{G, G^{\prime}}=\frac{2 m_{G, i j} m_{G^{\prime}, i j}+c}{m_{G, i j}^{2} m_{G^{\prime}, i j}^{2}+c},
$$

where $m_{G, i j}$ and $m_{G^{\prime}, i j}$ denote the gradient magnitudes of $G$ and $G^{\prime}$ at $(i, j)$, respectively, and $c$ is a positive constant for numerical stability. A lower value of GMSD means higher quality. The MATLAB source code for calculating GMSD can be downloaded at http://www4.comp.polyu.edu.hk/ cslzhang/IQA/GMSD/G MSD.htm.

\subsection{EMEG over GMSD}

EMEG/GMSD proposed by Yu et al. ${ }^{(3,5)}$ incorporates the above EMEG and GMSD into a single value as follows:

$$
\operatorname{EMEG} / \operatorname{GMSD}\left(G, G^{\prime}\right)=\frac{\operatorname{EMEG}\left(G^{\prime}\right)}{\operatorname{GMSD}\left(G, G^{\prime}\right)}
$$

The larger the value of EMEG/GMSD is, the higher the image quality. This property of EMEG/GMSD is consistent with that of both EMEG and GMSD.

\section{Experimental Results}

Fig. 1 shows the results of contrast enhancement of a grayscale image 'Building' in the SIDBA standard image database $^{(8)}$. Fig. 1(a) shows the original image, and Figs. 1(b)-(e) show the contrast-enhanced images by HE, linear, constant and the proposed HSs, respectively. Both Figs. 1(c) and (d) are over-enhanced, and show the intensity saturation on the holy wall and the leaves of trees. Fig. 1(e) shows the output image of the proposed HS. In Fig. 1 (e), the overenhancement observed in Figs. 1(c) and (d) is suppressed.

Fig. 2 shows the histograms corresponding to the images in Fig. 1. In each graph in Fig. 2, the vertical and horizontal axes denote the number of pixels and the intensity, respectively. In Fig. 2(b), we can see many empty or 0valued bins. The linear, constant and proposed HS methods try to fill the empty bins in the equalized histogram with neighboring non-zero values to obtain the target histogram. The bright regions in Figs. 1(c) and (d) increase the values in the histograms in Figs. 2(c) and (d) at the interval between 236 and 254 on the horizontal axes, and distort the shape of the original histogram in Fig. 2(a). On the other hand, in Fig. 2(e) which shows the histogram obtained by the proposed HS method, the shape of the original histogram (a) is preserved

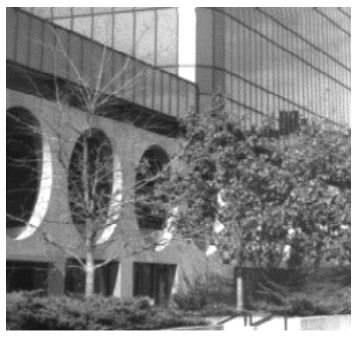

(a) Original.

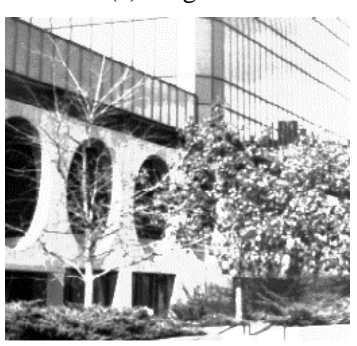

(c) Linear HS.

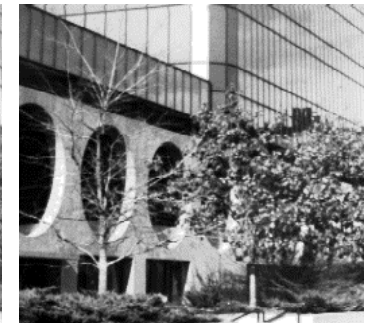

(b) HE.

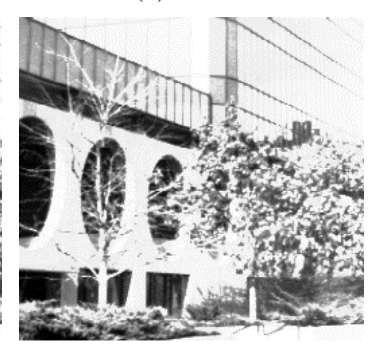

(d) Constant HS.

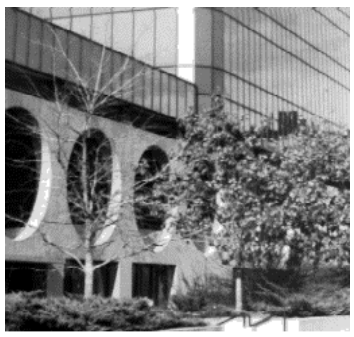

(e) Proposed HS.

Fig. 1. Results of contrast enhancement.

well. Figs. 3(a)-(c) show the target histograms for the linear, constant and proposed HS methods, respectively, where green lines denote the equalized histogram in Fig. 2(b), and red lines denote the interpolated target histograms. In Figs. 3(a) and (b), linear and constant HS produce very different interpolated results at the interval $[236,254]$ on the horizontal axes. This kind of modification of the shape of histogram causes contrast over-enhancement as shown in Figs. 1(c) and (d). On the other hand, the proposed method provides a reasonable interpolation result as shown in Fig. 3(c). As a result, the over-enhancement is avoided in Fig. 1(e).

The results of contrast enhancement for other images are shown in Fig. 4, where the 11 original images are shown in the top row, and the corresponding output images by HE, linear, constant and proposed HS methods are shown from the second to fourth rows, respectively. We can see the contrast over-enhancement and its suppression similar to that in Fig. 1 for two images 'Lighthouse' and 'Text' in Fig. 4. 


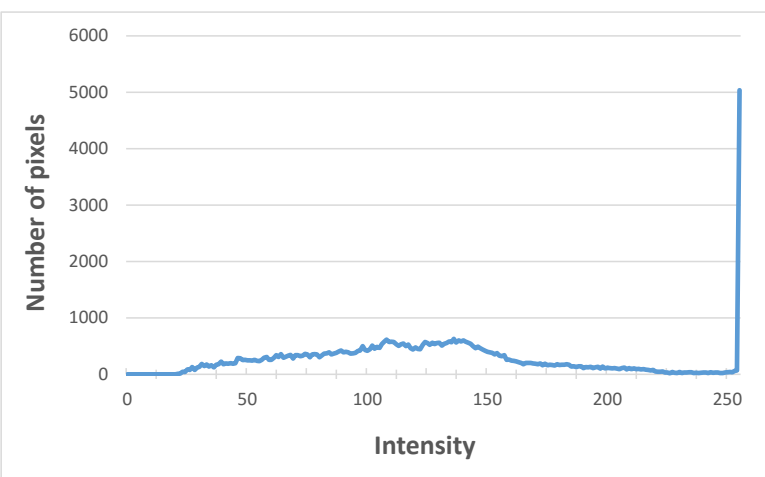

(a) Original.

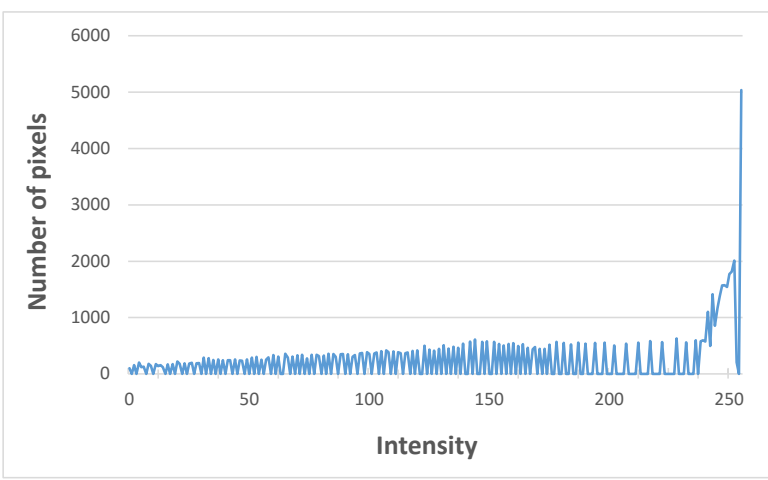

(c) Linear HS.

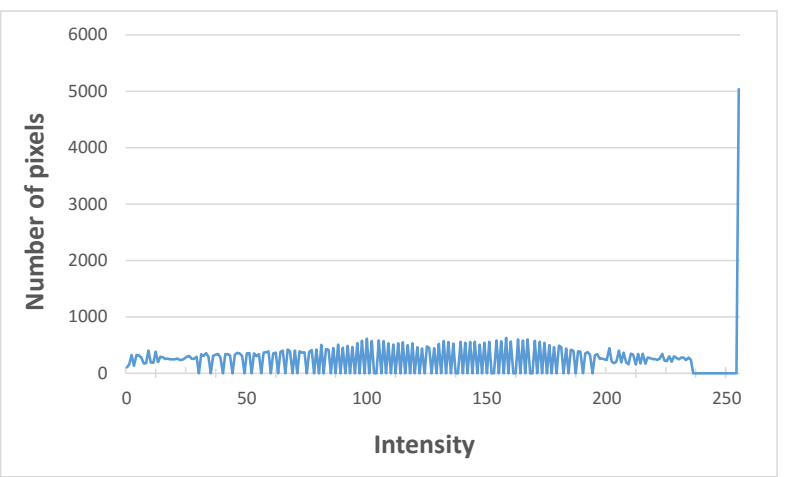

(b) HE.

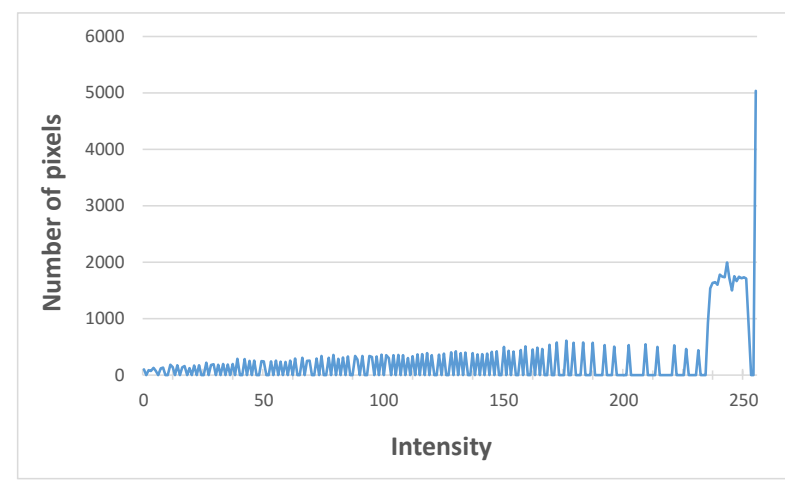

(d) Constant HS.

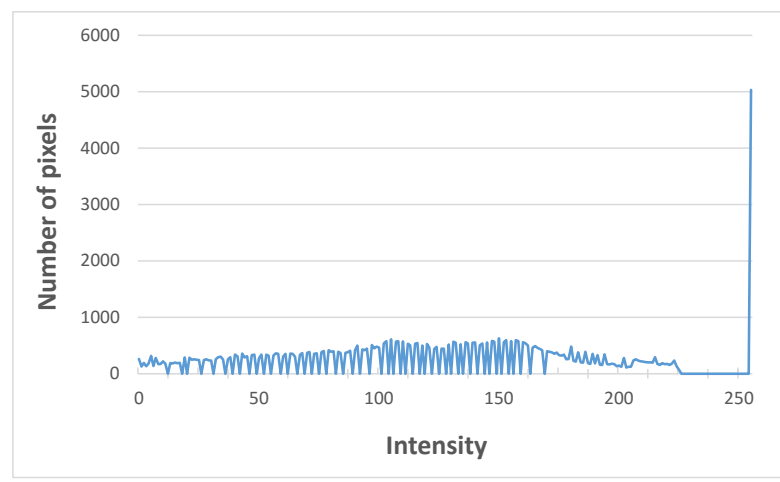

(e) Proposed HS.

Fig. 2. Histograms of images in Fig. 1.

Table 1 shows the evaluated values of the three image quality measures, EMEG, GMSD and EMEG/GMSD (E/G), for the above enhanced images. For each image and each image quality measure, four contrast enhancement methods, $\mathrm{HE}$, linear, constant and proposed HS, are compared, and the best performance among the four methods is indicated by bold numbers in Table 1. The bottom row in the table shows the average values of the 12 images. For EMEG, HE achieved the highest value on average. For GMSD, linear HS achieved the lowest value on average. For E/G, constant HS achieved the highest value on average. The proposed method achieved the best GMSD and E/G values for two images
'Building' and 'Lighthouse'. Among the three image quality measures, EMEG has a tendency to prefer HE since HE achieved the highest average value. GMSD and E/G tend to select similar images and methods as good qualities, and assign better values to 'Building' and 'Lighthouse' images enhanced by the proposed HS. However, for 'Text' image, the proposed HS received a low evaluation, although the enhanced images by $\mathrm{HE}$ and the proposed HS are visually similar. These observations suggest that the three image quality measures are not almighty ones for objective evaluation of image quality. 


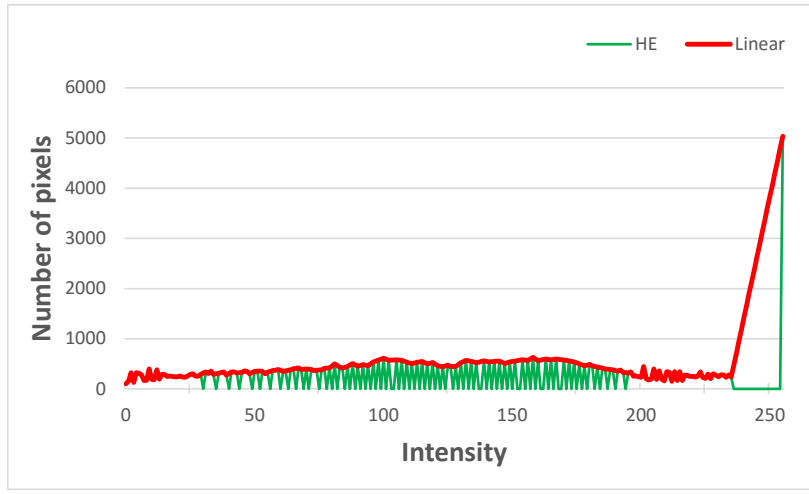

(a) Linear HS.

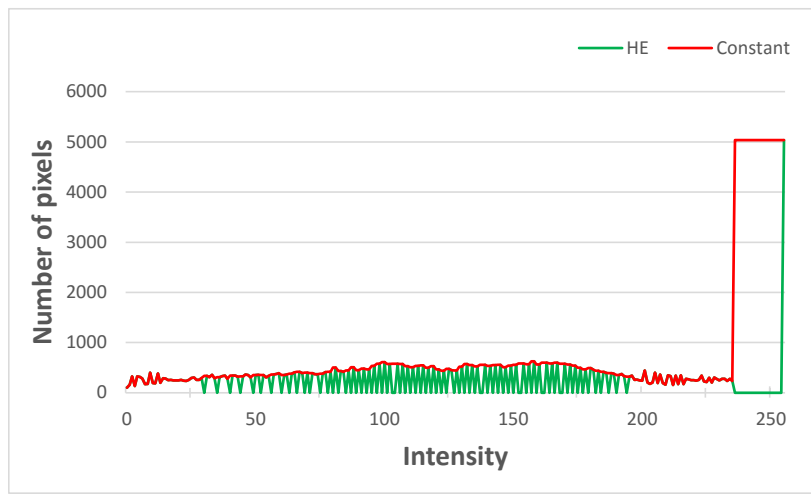

(b) Constant HS.

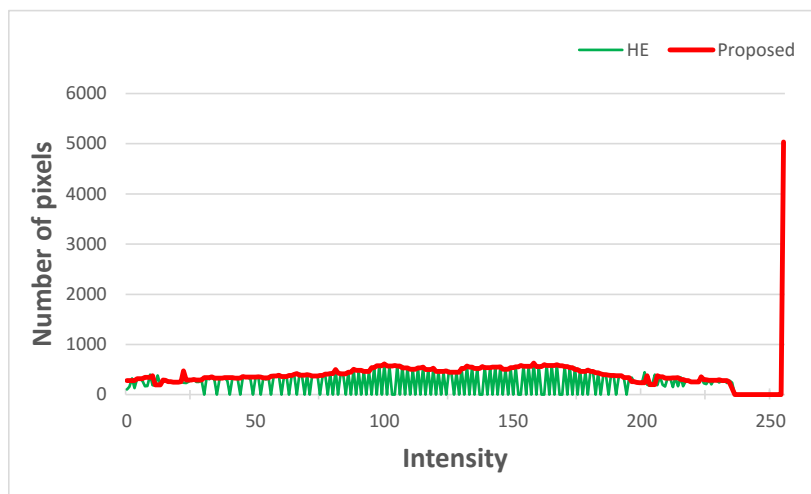

(c) Proposed HS.

Fig. 3. Target histograms.

\section{Conclusions}

In this paper, we proposed a method for image contrast enhancement by a histogram specification (HS) method where the target histogram for a given grayscale image is automatically computed from the given image by using local maximum and minimum selection operations. The target histogram is obtained by interpolating the equalized histogram of a given image by filling its empty bins with the neighboring non-zeros values. Experimental results show that the proposed is also effective for some images which are over-enhanced by previous HS methods. Future work will include the development of image quality measures consistent with the image quality evaluation by human beings.

\section{Acknowledgments}

This work was supported by JSPS KAKENHI Grant Number JP16H03019.

\section{References}

(1) R. C. Gonzalez and R. E. Woods : Digital Image Processing, Pearson Prentice Hall, 3rd ed., 2008

(2) R. P. Singh, M. Dixit : "Histogram Equalization: A Technique for Image Enhancement”, International Journal of Signal Processing, Image Processing and Pattern Recognition, Vol. 8, No. 8, pp. 345-352, 2015

(3) H. Yu, K. Inoue, K. Hara, K. Urahama : "Image Contrast Enhancement by Stepwise Histogram Specification”, Journal of Institute of Industrial Applications Engineers, Vol. 3, No. 4, pp. 161-166, 2015

(4) M. Nikolova and G. Steidl : "Fast Ordering Algorithm for Exact histogram Specification”, IEEE Trans. Image Process., Vol. 23, No. 12, pp. 5274-5283, 2014

(5) H. Yu, K. Inoue, K. Hara, K. Urahama : "Image Contrast Enhancement by Interpolative Histogram Specification”, IEICE technical report, Vol. 115, No. 459, pp. 91-96, 2016 (in Japanese)

(6) T. Celik : "Spatial Entropy-Based Global and Local Image Contrast Enhancement”, IEEE Trans. Image Process., Vol. 23, No. 12, pp. 5298-5308, 2014

(7) W. Xue, L. Zhang, X. Mou, A. C. Bovik : “Gradient Magnitude Similarity Deviation: A Highly Efficient Perceptual Image Quality Index”, IEEE Trans. Image Process., Vol. 23, No. 2, pp. 684-695, 2014 

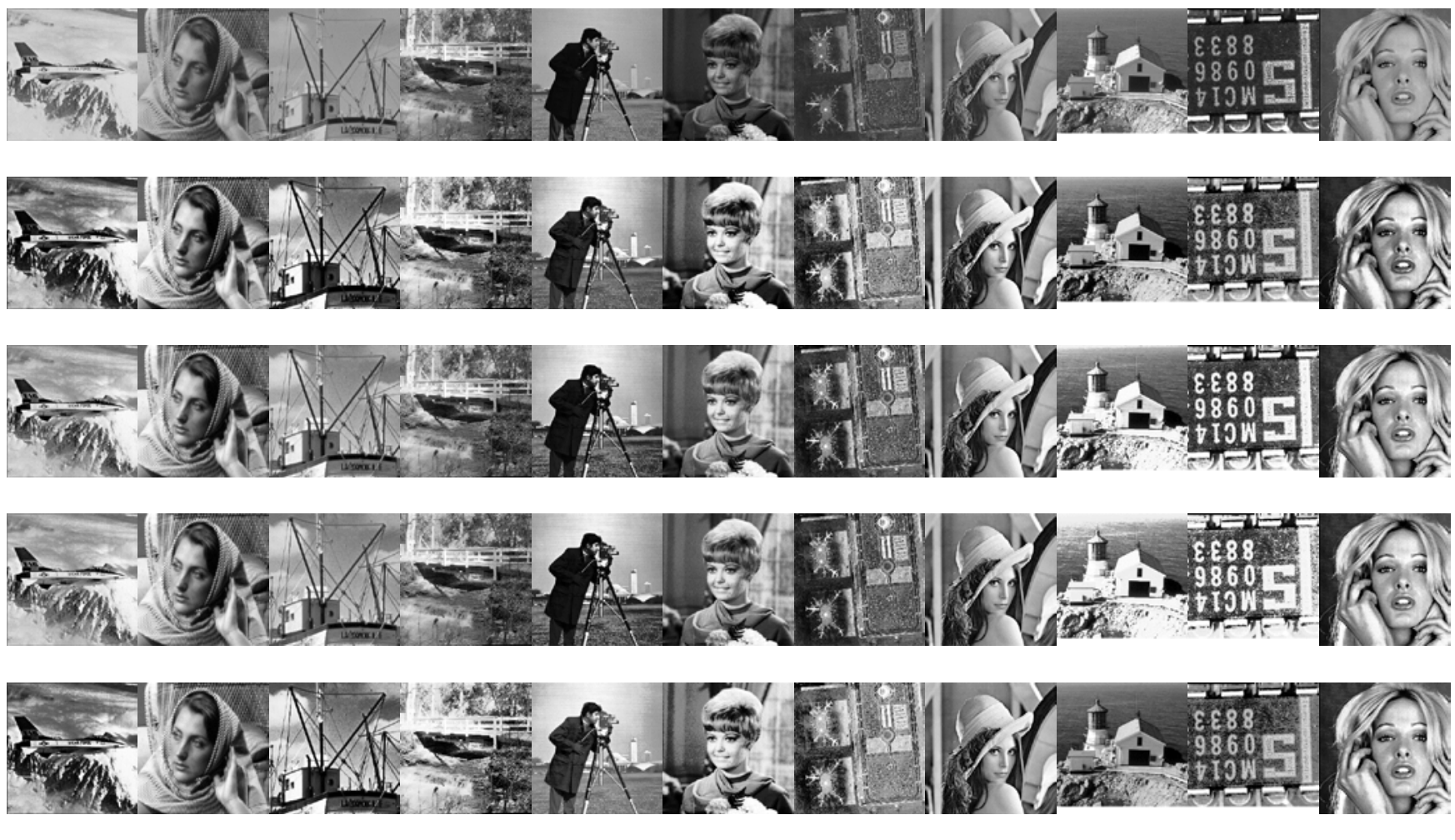

Airplane Barbara Boat Bridge Cameraman Girl Lax Lena Lighthouse Text Woman

Fig. 4. Contrast enhancement results. From top to bottom rows, the original images, their output images by HE, linear, constant and proposed HS methods are shown, respectively.

Table 1 . The values of image quality measures.

\begin{tabular}{|c|c|c|c|c|c|c|c|c|c|c|c|c|}
\hline & \multicolumn{4}{|c}{ EMEG } & \multicolumn{4}{|c|}{ GMSD } & \multicolumn{4}{c|}{ E/G } \\
\cline { 2 - 13 } & HE & Linear & Const. & Prop. & HE & Linear & Const. & Prop. & HE & Linear & Const. & Prop. \\
\hline Airplane & 0.3782 & 0.2611 & 0.2618 & $\mathbf{0 . 3 8 3 4}$ & 0.2079 & $\mathbf{0 . 1 1 4 9}$ & 0.1169 & 0.2353 & 1.8191 & $\mathbf{2 . 2 7 2 7}$ & 2.2403 & 1.6292 \\
\hline Barbara & $\mathbf{0 . 2 7 4 0}$ & 0.255 & 0.255 & 0.2547 & 0.0404 & $\mathbf{0 . 0 2 2 1}$ & 0.0222 & 0.0228 & 6.7873 & $\mathbf{1 1 . 5 4 0}$ & 11.48 & 11.181 \\
\hline Boat & 0.4182 & 0.2804 & 0.2818 & $\mathbf{0 . 4 3 9 4}$ & 0.2119 & $\mathbf{0 . 1 3 5 6}$ & 0.1367 & 0.2336 & 1.9736 & $\mathbf{2 . 0 6 6 9}$ & 2.0618 & 1.8809 \\
\hline Bridge & 0.4612 & 0.3704 & 0.3721 & $\mathbf{0 . 5 1 1 0}$ & 0.0556 & 0.0112 & $\mathbf{0 . 0 0 9 6}$ & 0.0996 & 8.2985 & 33.155 & $\mathbf{3 8 . 7 6 4 6}$ & 5.1308 \\
\hline Building & 0.3194 & 0.3693 & $\mathbf{0 . 3 7 0 5}$ & 0.2702 & 0.0738 & 0.1499 & 0.1734 & $\mathbf{0 . 0 4 4 0}$ & 4.3281 & 2.4628 & 2.1368 & $\mathbf{6 . 1 4 7 3}$ \\
\hline Cameraman & 0.2808 & 0.3111 & $\mathbf{0 . 3 1 3 0}$ & 0.2592 & 0.1167 & $\mathbf{0 . 1 1 4 0}$ & 0.1150 & 0.1519 & 2.4069 & $\mathbf{2 . 7 3 0 1}$ & 2.7216 & 1.7067 \\
\hline Girl & 0.2466 & 0.1938 & 0.1939 & $\mathbf{0 . 2 6 2 8}$ & 0.1409 & 0.0602 & $\mathbf{0 . 0 5 9}$ & 0.1758 & 1.7497 & 3.2189 & $\mathbf{3 . 2 8 4 6}$ & 1.4947 \\
\hline Lax & $\mathbf{0 . 6 4 7 3}$ & 0.5632 & 0.5648 & 0.6222 & 0.1619 & $\mathbf{0 . 0 6 7 1}$ & 0.0669 & 0.1899 & 3.9986 & 8.3901 & $\mathbf{8 . 4 3 7 4}$ & 3.2768 \\
\hline Lena & $\mathbf{0 . 2 5 3 7}$ & 0.2295 & 0.2295 & 0.2317 & 0.0650 & $\mathbf{0 . 0 3 3 0}$ & 0.0332 & 0.0348 & 3.9062 & $\mathbf{6 . 9 4 6 6}$ & 6.9102 & 6.6646 \\
\hline Lighthouse & 0.3848 & 0.4220 & $\mathbf{0 . 4 3 7 2}$ & 0.3234 & 0.090 & 0.1727 & 0.2189 & $\mathbf{0 . 0 4 1 3}$ & 4.2736 & 2.4432 & 1.9978 & $\mathbf{7 . 8 3 3 0}$ \\
\hline Text & 0.4101 & 0.5037 & $\mathbf{0 . 6 0 5 3}$ & 0.3901 & $\mathbf{0 . 1 7 1 6}$ & 0.1748 & 0.2636 & 0.1860 & 2.3896 & $\mathbf{2 . 8 8 1 4}$ & 2.2965 & 2.0969 \\
\hline Woman & $\mathbf{0 . 2 8 6 9}$ & 0.2690 & 0.2721 & 0.2692 & 0.1171 & $\mathbf{0 . 0 6 9 2}$ & 0.0704 & 0.0699 & 2.4506 & $\mathbf{3 . 8 8 8 6}$ & 3.8655 & 3.8530 \\
\hline Average & $\mathbf{0 . 3 6 3 4}$ & 0.3357 & 0.3464 & 0.3514 & 0.1211 & $\mathbf{0 . 0 9 3 7}$ & 0.107 & 0.1237 & 3.6985 & 6.8330 & $\mathbf{7 . 1 8 3 3}$ & 4.4079 \\
\hline
\end{tabular}

(8) M. Sakauchi, Y. Ohsawa, M. Sone, M. Onoe :

"Management of the standard image database for image processing researches”, ITEJ Technical Report, Vol. 8, No. 38, pp. 7-12, 1984 (in Japanese) 